\title{
La inserción de Facebook en actividades académicas*
}

\author{
Rosalba Rojas Balaguera*
}

Fecha de recepción: 6 de julio de 2012

Fecha de revisión: 27 de agosto de 2012

Fecha de aprobación: 30 de octubre de 2012

\section{Resumen}

El estudio aborda la temática relacionada con la socialización del conocimiento disciplinar en ambientes mediados por tecnología, para hacer un análisis del uso de Facebook por parte de los estudiantes, a fin de generar un diagnóstico sobre su utilización en actividades académicas. Toma como marco de referencia el enfoque sociocultural de Vigotsky y los estudios realizados por James (2004), así como autores que destacan la importancia de involucrar en los procesos de aprendizaje los intereses de los alumnos. El estudio ofrece una alternativa para aquellos profesores que consideran la importancia de las tecnologías y las preferencias de los estudiantes en los procesos de enseñanza-aprendizaje.

Palabras clave: práctica social, Facebook, saberes disciplinares, apropiación y dominio, redes sociales, conocimiento, medios virtuales e internet.

* $\quad$ Artículo de investigación científica y tecnológica que presenta los resultados originales del proyecto titulado "Facebook como apoyo en actividades académicas para la apropiación de saberes disciplinares", lidearado por la Magíster en Educación Rosalba Rojas.

** Máster en Tecnología Educativa y Medios Innovadores para la Educación, funge como docente en la Institución Educativa Héctor Julio Rangel Quintero del municipio de Floresta, Boyacá, Colombia, sede San Luis. Realiza la investigación titulada "Facebook como apoyo en actividades académicas para la apropiación de saberes disciplinares". Correo electrónico: rosalba.rojas9@gmail. com 


\title{
The inclusion of Facebook in academics
}

\begin{abstract}
This study addresses the issues related to the socialization of disciplinary knowledge in technology-mediated environments to make an analysis of the use of Facebook by students, in order to assessits use in academic activities. We take as reference Vygotsky's sociocultural approach and studies by James (2004), as well as of other authors, who stress the importance of engaging students' interests in the learning process. The study offers an alternative for those teachers who consider the importance of technologies and the preferences of students in the teaching-learning process.
\end{abstract}

Keywords: social practice, Facebook, disciplinary knowledge, ownership anddomain, social networks, knowledge, virtual media and internet.

\section{A inserção do Facebook nas atividades acadêmicas}

\section{Resumo}

O estudo aborda as questões relacionadas com a socialização do conhecimento disciplinar em ambientes mediados pela tecnologia, para fazer uma análise da utilização do Facebook pelos alunos, a fim de gerar um diagnóstico sobre seu uso nas actividades académicas. Tomando como marco de referência a abordagem sociocultural de Vygotsky e os estudos realizados por James (2004), assim como autores que enfatizam a importância de se envolver nos processos de aprendizagem dos interesses dos estudantes. O estudo oferece uma alternativa paraa que les professores que consideram a importância das tecnologiase as preferências dos alunos nos processos de ensino-aprendizagem

Palavras-chave: prática social, Facebook, saberes disciplinares, apropriação e domínio, redes sociais, conhecimento, médios virtuais e internet. 


\section{Introducción}

El estudio aborda la temática relacionada con la socialización del conocimiento disciplinar en ambientes mediados por tecnología digital, para hacer un análisis acerca del uso de la red social Facebook por parte de los estudiantes, a fin de generar un diagnóstico sobre su aplicación en actividades académicas. Además, ofrece una visión al panorama que se presenta ante la inserción de los medios virtuales dentro de la cultura de las personas y el surgimiento de las nuevas tecnologías, estableciendo una estrecha relación con el enfoque sociocultural de Vigotsky y los estudios realizados por James (2004) y otros autores en cuanto a la importancia de involucrar dentro de los procesos de aprendizaje, los intereses de los niños y de los jóvenes, especialmente en lo relacionado con la mediación de tecnología digital.

\section{Enfoque metodológico de la investigación}

El enfoque metodológico utilizado en la investigación para recolectar la información fue de carácter cualitativo, el cual permite el desarrollo de estrategias como la observación participante y la entrevista cualitativa de tipo semiestructurado, a partir de las que se realiza la recolección de datos con el fin de definir las propiedades y características del contexto en el que se desenvuelve la población de muestra. Según Vela (2004), la metodología en mención permite analizar el significado de las relaciones sociales, que en este caso se consideran las llevadas a cabo entre un grupo de veinticinco estudiantes del grado décimo a través de la red social Facebook.

Con el análisis de los resultados se encuentra que los alumnos interactúan con frecuencia en Facebook, pero que a pesar del uso de las tecnologías de la información y la comunicación en los diferentes campos de la vida cotidiana, aún no se desarrollan actividades académicas con prácticas que involucren la utilización de sus herramientas en las mismas. Este estudio ofrece como alternativa la implementación de la red en mención en los procesos de enseñanza aprendizaje, de tal forma que se tengan en cuenta las preferencias de los estudiantes durante la orientación disciplinar.

\section{¿Por qué usar Facebook en actividades escolares?}

Con la aparición de las nuevas tecnologías se hace necesario que las personas adquieran habilidades que le permitan ser competentes en los diferentes contextos. Razón por la que es preciso revisar hasta qué punto los estudiantes implementan las experiencias de su vida cotidiana en los procesos de apropiación y dominio de los saberes disciplinares, en especial en lo referente al uso de las herramientas de la red social Facebook, teniendo en cuenta que es común escuchar entre los jóvenes la expresión "si no tienes Face no existes".

En este orden de ideas, es conveniente reflexionar sobre los planteamientos del enfoque sociocultural de Vigotsky (citado en Fernández, 2009b), quien afirma: 
El desarrollo humano y el aprendizaje no son construcciones individuales, sino que son fundamentalmente el producto de la participación social ligado al uso de herramientas culturales (Fernández, 2009b, p. 15).

La inclusión de las Tecnologías de la Información y la Comunicación (TIC) ha permitido la creación de espacios virtuales en los que los estudiantes pueden compartir información como documentos, videos, fotos y algunos medios de comunicación inmediata, como el chat, entre otros; los cuales pueden ser de gran ayuda en el momento de desarrollar los procesos de construcción de conocimiento, pero si no se utilizan adecuadamente pierden su esencia.

Sin embargo, en algunas instituciones educativas colombianas -para este caso en particular, en la sección de secundaria de la Institución Educativa Héctor Julio Rangel Quintero-a pesar de los adelantos tecnológicos, virtuales y de la aparición de los nuevos métodos que se pueden usar en la apropiación y dominio de los saberes disciplinares, aún no se ha realizado una indagación sobre el uso que hacen los alumnos de la red social Facebook, así como de la manera en la que dicha plataforma puede contribuir en los procesos de construcción de conocimiento.

Con el desarrollo de la presente investigación se ofrece a la comunidad de la institución educativa antes mencionada un diagnóstico para saber cuál es el uso que hacen de la red Facebook los estudiantes del grado décimo y si este está relacionado con los procesos de apropiación y dominio de los saberes disciplinares.
Con la inserción de las nuevas estrategias resultan beneficiados todos los miembros de la comunidad educativa, ya que se propone el desarrollo de actividades de aprendizaje a partir del uso de las herramientas de Facebook, a fin de alcanzar una mayor motivación por parte de los estudiantes en sus procesos académicos.

\section{Rompiendo paradigmas en las prácticas de construcción de conocimiento}

El estudio se plantea dentro del marco de las prácticas sociales emergentes de la sociedad de la información y su impacto en la educación, por tratar aspectos relacionados con Facebook, red en la que se puede compartir información de diferentes temáticas, a partir del uso de las herramientas que han surgido por la utilización de internet. La pregunta de investigación pretende indagar si los estudiantes utilizan este recurso dentro de los procesos de apropiación y dominio de los saberes disciplinares y si estas influyen en el desarrollo de las actividades académicas.

Teniendo en cuenta los planteamientos de la perspectiva sociocultural de Vigotsky -en lo relacionado con la inserción de las experiencias del contexto en los procesos de enseñanza aprendizaje y los estudios realizados por James (2004), en cuanto a la importancia de involucrar dentro de los procesos de aprendizaje los intereses de los niños y jóvenes en las actividades de socialización de conocimiento disciplinar, especialmente en lo relacionado con 
la mediación de tecnología digital-, se planteó la investigación partiendo de la pregunta:

¿De qué manera utilizan y cómo influye el uso de la red social Facebook en los procesos de apropiación y dominio de los saberes disciplinares, durante el desarrollo de las actividades académicas de los alumnos del grado décimo - uno de la institución educativa Héctor Julio Rangel Quintero del municipio de Floresta, Boyacá, Colombia?

\section{Aplicación de la tecnología en los procesos de enseñanza aprendizaje}

En la actualidad, como producto del avance tecnológico durante el proceso histórico evolutivo del hombre, la sociedad presenta una gran cantidad de innovaciones que ponen a disposición de la humanidad distintos tipos de herramientas digitales como los ordenadores y los celulares, entre otros, que cumplen la función de mediadores en las diferentes actividades que las personas desarrollan en su vida cotidiana.

En el proceso de investigación se abordan temáticas relacionadas con la utilización de los medios tecnológicos en la enseñanza de las diferentes disciplinas, de manera específica aquellos temas que tienen que ver con la participación de las personas en las comunidades de práctica.

Asimismo, el estudio revisa conceptos como la socialización y participación legítima periférica o manera mediante la cual se comparte un conocimiento de un campo específico, lo cual, en palabras de Martínez (2008), es un proceso que realiza un individuo de forma conjunta para adquirir un conocimiento. A su vez, la participación legítima periférica habla de las relaciones que se establecen entre novatos y veteranos (Wenger, 1991). Además, dentro de este proceso cobran importancia los aspectos relacionados con las comunidades de práctica, donde el conocimiento es construido por el propio aprendiz mediante las experiencias que vive en su grupo social, con prácticas de aprendizaje dadas a partir de la actividad de las personas dentro de un contexto y una cultura con una enseñanza situada y basada en la experiencia (Barriga, 2003).

Ahora bien, en lo que atañe a la relación con paradigmas y disciplinas académicas, son analizadas desde las perspectivas sociológica, antropológica y lingüística, bajo los estudios realizados por diferentes investigadores, quienes a través de sus planteamientos establecen relación entre los procesos de construcción de conocimiento y la evolución de la ciencia. Para Haspelmath (2001), el desarrollo de las teorías lingüísticas tiene un lugar especial en el proceso evolutivo de la ciencia, porque trata con el lenguaje, el cual requiere ser entendido como un tema para ser estudiado en sí mismo. Corvi (1996), en sus planteamientos, afirma que Popper en el transcurso de sus investigaciones trató sobre algunos aspectos de la psicología del conocimiento y el descubrimiento.

Por su parte, Haspelmath (2001) considera que el desarrollo de las teorías lingüísticas 
tiene un espacio central en la evolución de la ciencia, teniendo en cuenta que la lengua a través de la historia se ha convertido en el medio más importante de la comunicación humana. Moreno (2005) sostiene que la sociología de la educación ha hecho significativas aportaciones durante el transcurso de la evolución y la historia de la ciencia, mientras que Copertari (2009) destaca la importancia delsurgimiento de la educación virtual. Kuhn (1962) establece relación entre la revolución científica y los fenómenos de los paradigmas. De igual manera, se presentan planteamientos de Fernández (2009a) en cuanto a los paradigmas que abordan la inclusión de programas computacionales a través de la historia. Además, se integra el estudio realizado por Goodiwn (1997) sobre la implicación de diferentes fenómenos y procesos heterogéneos en la actividad cognitiva humana.

En lo concerniente al tema de la mediación tecnológica se hace énfasis en la perspectiva sociocultural de Vigotsky, en lo relacionado con tecnología, lengua escrita, cultura y educación y en las aplicaciones de la teoría sociocultural y de la actividad basada en los planteamientos de autores como Pinaya (2005), quien considera que es necesario implementar una reforma educativa hacia las prácticas de aula, con orientación constructivista en la que se dé importancia a la dimensión social en el desarrollo de los procesos psicológicos de los seres humanos y la forma en que el lenguaje afecta el desarrollo pedagógico. De igual manera, se analiza el punto de vista de Salmerón (2010), quien afirma que dentro de los principales avances en los modelos educativos se destacan los re- lacionados con la incorporación de tecnologías de la información y la comunicación. Además, se presentan los estudios de Sancho (2006) sobre el uso de la tecnología para transformar la educación.

Asimismo, se dan a conocer las investigaciones hechas por Fernández (2009b), quien muestra la importancia de las tecnologías de la información y la comunicación como instrumentos mediadores dentro de los procesos de aprendizaje, al igual que las indagaciones de La casa (2002) sobre el papel de la cultura en los procesos de interacción del individuo con el mundo. Además, se tienen en cuenta las propuestas de Daniels (2003) en lo relacionado con la aplicación educativa de la teoría sociocultural de Vigotsky; las propuestas de Hota (2000), en cuanto a la importancia del computador como instrumento mediador para facilitar el desarrollo de algunos trabajos del hombre, así como la revisión de las propuestas de Moll (1990) en lo relacionado con la aplicación de los nuevos medios de comunicación en la educación y la investigación.

La investigación profundiza la temática de los saberes disciplinares específicos, en cuanto a su socialización con la presentación de los planteamientos de diferentes autores como Cassany (1994), quien considera que el aprendizaje de la lengua se da mediante la adquisición y el desarrollo de estrategias que permitan comunicarse dejando de lado las prácticas individuales de los alumnos y el papel protagónico del maestro. De igual forma, se muestran los planteamientos de Cole (2003) en los que destaca el desarrollo de la mente 
humana en relación con la evolución de la cultura. Por otra parte, se analizan las propuestas de Freire (2005) en su investigación basada en la necesidad de implementar propuestas pedagógicas que permitan alcanzar la liberación del hombre, teniendo en cuenta que dentro de la sociedad los individuos se presentan como opresores u oprimidos.

También se destacan las investigaciones de La Casa (1988), quien propone un estudio basado en las perspectivas de Piaget y Vigotsky, para ofrecer unos mejores entornos de aprendizaje al niño mediante la interacción social. De igual forma, se fundamenta en los análisis hechos por Lillis (2003), en sus estudios relacionados con los contextos y los diferentes grupos sociales en que se desarrolla el aprendizaje de un idioma, a partir de la perspectiva sociocultural, la cual afirma que las personas aprenden por la interacción que se da dentro su medio. Asimismo, se tienen en cuenta los estudios realizados por Kress, Jewitt, Ogborn y Tsatsarelis (2001) en cuanto a la perspectiva multimodal que considera que existen diferentes formas de comunicación, entre las que mencionan el lenguaje oral, el escrito y el visual, los cuales permiten dar significado a los aprendizajes.

En esta investigación se consideran además los planteamientos de Suchman (2007) sobre la interacción que se da entre el hombre y la máquina, los problemas que se pueden presentar en la construcción de máquinas inteligentes interactivas y el uso de los recursos tecnológicos en el momento de desarrollar un propósito. Asimismo, se presentan las investigaciones de James (2004) hechas en su crítica al uso del lenguaje en la escuela tradicional y su propuesta sobre una alternativa de aprendizaje en la que se tienen en cuenta los intereses de los niños y jóvenes, como por ejemplo el uso de video-juegos en los procesos de construcción de conocimiento.

Es importante reconocer la necesidad de la interacción de las personas, en tanto que el ser humano es sociable por naturaleza y desde sus primeras experiencias adquiere el aprendizaje en un mundo compartido en situaciones determinadas. Barriga (2003) sostiene que los principios de la cognición situada propuestos dentro del saber sociocultural de Vigotsky, consideran que el conocimiento forma parte y es producto de una actividad cuando se da a partir de las interacciones de las personas dentro de un contexto y una cultura con una enseñanza basada en la práctica, como por ejemplo, la solución de problemas, experiencias de vida, análisis de casos y desarrollo de proyectos, entre otros. Por su parte, Moreno (2005) hace un aporte al tema de la perspectiva sociocultural al afirmar que para algunos pensadores como Durkheim, la educación desde la perspectiva funcionalista es una variable dependiente de lo social; según este pensador, la escuela se convierte en la forma más adecuada para favorecer la integración de los individuos a su medio sociocultural.

Un concepto relacionado con la perspectiva sociocultural de Vigotsky es el de la participación legítima periférica, la cual, según Wenger (1991), adquiere su definición de interconexiones a partir de "personas, actividades, conocimientos y mundo" (Wenger, 1991, p. 46). De 
tal forma que da importancia al significado del aprendizaje basado en la experiencia del ser humano, que es concebido como miembro de una comunidad en la que sufre un proceso de transformación de novato a practicante, para llegar a convertirse en veterano como una parte integral de la acción de su conocimiento.

Otro aspecto por destacar es el concerniente a las indagaciones de Barriga (2003) en lo relacionado con los principios de la cognición situada, propuestos dentro del saber sociocultural de Vigotsky, los cuales sostienen que el conocimiento es situado cuando se da a partir de la actividad de las personas dentro de un contexto y una cultura con una enseñanza basada en la práctica, como por ejemplo la solución de problemas, experiencias de vida, análisis de casos y desarrollo de proyectos, entre otros.

Los estudios realizados en cuanto a la historia de la perspectiva sociocultural destacan los aportes de Cole (2003), quien sostiene que con la evolución de la humanidad han avanzado también las relaciones entre mente y cultura. En este campo se han hecho investigaciones que defienden los planteamientos de la perspectiva sociocultural, como los realizados por La Casa (1988) en lo relacionado con el desarrollo cognitivo de las personas, poniendo especial atención a la forma en que el niño va tomando conciencia de sí mismo y de su propia acción dentro de la interacción social y del papel que desempeña el lenguaje en el desarrollo del conocimiento.
Años más tarde, Moll (1990) presenta una propuesta en la que el enfoque sociocultural de Vigotsky se caracteriza por tratar temas relacionados con el análisis genético para el desarroIlo; la afirmación de las funciones mentales más altas en lo individual y sus orígenes en la vida social; la ratificación de una clave esencial para entender los procesos psicológicos humanos y sociales a partir de herramientas y señales utilizadas para mediar el uso del análisis genético y el funcionamiento mental humano que para él significa la ruta principal para comprender la mente.

Por su parte, Goodiwn (1997) presenta una visión de la naturaleza social del lenguaje como un sistema compartido de significados preestablecidos y una estructura que tiene consecuencias mucho más allá de la lingüística. Para explorar cómo se utiliza el lenguaje en un proceso, este se debe examinar en una situación de trabajo específico. Afirma que es necesario que los interlocutores cuando usen un término adecuado a un contexto determinado sean co-partícipes de una actividad para lograr comprender el mensaje que se da, bien sea de forma oral o gestual. Es a lo que Vigotsky denomina una zona de desarrollo próxima.

Asimismo, se han adelantado propuestas que establecen relación entre la perspectiva sociocultural y las formas de comunicación que se dan entre los individuos. Haspelmath (2001) considera que la lingüística es un tema relacionado con el estudio del lenguaje humano; el cual cuenta con diferentes subdivisiones en distintas ramas y tiene diversas relaciones con otras disciplinas. Explica que la historiografía 
lingüística es la encargada de analizar su desarrollo y tiene como finalidad aclarar las líneas de su proceso en entornos particulares, al igual que su interacción con otras disciplinas.

Por otro lado, La Casa (2002) plantea que el lenguaje es el elemento de comunicación más importante de los seres humanos, a partir del cual el hombre ha alcanzado un desarrollo evolutivo a través de la historia con el uso de "instrumentos materiales, signos y sistemas simbólicos" (La Casa, 2002, p. 28). Estos se han convertido en el medio por el que la actividad humana cobra sentido dentro de la naturaleza, en donde los instrumentos tienen una función dirigida del sujeto al objeto y el signo hacia el dominio del individuo mismo.

En investigaciones más recientes, como las realizadas por Fernández (2009b), se concluye que "el desarrollo humano y el aprendizaje no son construcciones individuales, sino que fundamentalmente son el producto de la participación social ligado al uso de herramientas culturales" (Fernández, 2009b, p. 15), con lo que muestra cómo las repuestas de un individuo surgen de la interacción en contexto.

Para Vigotsky (citado en Fernández, 2009b), "el desarrollo humano y el aprendizaje no son construcciones individuales, sino que son fundamentalmente el producto de la participación social ligada al uso de herramientas culturales" (Fernández, 2009b, p.15). Por otra parte, Pinaya (2005) afirma que ante la necesidad de implementar una reforma educativa en las prácticas de aula hacia una orientación constructivista, con mayor interacción entre los que participan en la actividad de construcción de conocimiento, se vienen realizando estudios apoyados en una "concepción de la actividad educativa como un acto de la naturaleza socio-histórica y cultural, que se desarrolla fundamentalmente dentro del aula como un espacio social de interacción e intercambio comunicativo" (Pinaya, 2005, p. 23). Además conlleva la apropiación y dominio de las diferentes ciencias las cuales se han agrupado en ramas del aprendizaje.

Para Iglesias (2007), "el saber disciplinar supone una estructuración y organización del conocimiento en relación con las disciplinas específicas" (Iglesias, 2007, p. 214). En esta medida, los conocimientos disciplinares hacen relación al conjunto de disciplinas científicas organizadas a partir de materias o asignaturas. Según Hernández (2002), estos "tienen importancia fundamental ya que ellos son los que nutren y dan lógica a los contenidos" (p. 19).

Por su parte, Pérez (2003) considera que se deben involucrar proyectos de investigación dentro de las prácticas escolares, como una alternativa de transformación en los diversos dominios disciplinares, integrando las diferentes actividades como estrategias de apropiación de la realidad, en la construcción de los procesos de enseñanza aprendizaje y de la interacción social.

Con el surgimiento de la tecnología digital se vienen implementando nuevas formas de interacción a partir del uso de las redes de la internet; según Bugeja (2006), estas son plataformas que han tenido gran influencia en la 
educación, porque ofrecen ventajas por ser un recurso para la comunicación global compartida, que al ser utilizadas permiten el desarrollo del pensamiento crítico. Además, las redes sociales promueven la interacción de una gran cantidad de personas y fomenta la organización $\mathrm{y}$ fortalecimiento de las comunidades virtuales.

Según Pardo (2010), Facebook es "un sistema operativo social" (p. 83), que más allá de los espacios conversacionales ofrece una transformación en las prácticas de alfabetización que permiten una digitalización de la identidad. A su vez, en los últimos años se ha convertido en una forma de vida en línea de muchas personas, ya que contiene un gran número de aplicaciones que se pueden utilizar, incluso como una cuenta de correo electrónico. De acuerdo con los estudios realizados por Pardo (2010), "según el ranking de Alexa de noviembre de 2009, ya ocupa el segundo lugar entre las plataformas más visitadas del mundo, por detrás de Google" (p. 84).

\section{Objetivos}

\section{Objetivo general}

Analizar el uso de la red Facebook por parte de los estudiantes del grado décimo - uno, de la institución educativa Héctor Julio Rangel Quintero del municipio de Floresta, Boyacá, Colombia, a fin de generar un diagnóstico sobre su utilización en actividades académicas, a partir de la aplicación de observaciones hechas al muro de cada estudiante (con su autorización) y del desarrollo de entrevistas que permitan indagar sobre su utilización y el tipo de interacciones que se desarrollan en este.

\section{Objetivos específicos}

- Identificar las diferentes herramientas de la red social Facebook utilizadas por los estudiantes del grado décimo - uno de la institución educativa Héctor Julio Rangel Quintero del municipio de Floresta, Boyacá, Colombia.

- Evaluar la utilidad de las actividades desarrolladas por los estudiantes de grado décimo - uno, de la institución educativa Héctor Julio Rangel Quintero del municipio de Floresta, Boyacá, Colombia en la red social Facebook.

- Determinar la posibilidad de utilizar las herramientas de Facebook en los procesos de construcción de conocimiento.

\section{Una mirada hacia la interacción de los jóvenes de hoy}

La metodología para el desarrollo del estudio sobre el uso de la red social Facebook, por parte de los estudiantes en la realización de actividades académicas, se basa en el enfoque cualitativo, porque este presenta características que permiten hacer una investigación sin medición de datos de forma numérica, de tal manera que posibilite centrar el interés de la indagación, sobre la actitud que asumen los alumnos del grado décimo - uno, de la institución educativa Héctor Julio Rangel Quintero del municipio de Floresta, frente a la utilización de las nuevas tecnologías y si estas 
son utilizadas en los procesos de apropiación y dominio de saberes disciplinares, teniendo en cuenta que el establecimiento educativo está dotado de una sala de informática que consta de quince computadores con acceso a la internet, en donde los estudiantes hacen uso de diferentes páginas.

Con este método se realizan descripciones minuciosas de diferentes eventos que se dan con el uso de dicho medio virtual, al aplicar entrevistas a los participantes en las que se puede ver el concepto de los alumnos con referencia al entorno en cuestión y la aplicación de observaciones pormenorizadas en los muros de cada uno de los integrantes; con el fin de esclarecer su verdadera utilidad e importancia, para hacer una propuesta sobre su inclusión dentro de las actividades académicas que permita alcanzar lo que según Duart (1999) se considera como cambios en las metodologías con la inclusión de los entornos virtuales en los procesos de construcción de conocimiento.

Según Hernández, Fernández y Baptista (2006), la recolección de los datos en un proceso cualitativo, no es estandarizada ni estadística; consiste en obtener las perspectivas y puntos de vista de los participantes a partir de una indagación subjetiva para reconocer tendencias personales. Además, el autor considera que este enfoque es flexible, tiene como propósito reconstruir la realidad tal como se observa, recibe también el nombre holístico por considerar el todo. No presenta manipulación de la realidad, está fundamentado en la interpretación de las acciones de los seres vivos y de sus interacciones en el medio, el cual se convierte en la fuente de datos.

Dentro del presente proceso de investigación se aplican entrevistas que permiten detectar los intereses de los alumnos, en cuanto al desarrollo de las actividades escolares y sus preferencias en lo relacionado con los ambientes de aprendizaje, así como el tiempo y los recursos que disponen para establecer interacciones con el uso de entornos virtuales y las dificultades que se presentan en los procesos por la falta de implementación de nuevos paradigmas en las prácticas educativas.

En la investigación participan 25 estudiantes integrantes del grado décimo - uno, de la institución educativa Héctor Julio Rangel Quintero del municipio de Floresta Boyacá, Colombia; quienes se encuentran en promedio de dieciséis a dieciocho años de edad y pertenecen a los estratos socioeconómicos uno, dos y tres; de familias que se dedican principalmente a la ganadería y a labores artesanales como la elaboración de alpargatas y el procesamiento de cueros, entre otras.

Según Mina (2004), "la estratificación adoptada en los distintos municipios del país incluye variables relacionadas con las características de la vivienda y su entorno" (p. 55). Para su realización se han tenido en cuenta ocho variables, a saber: existencia de viviendas a los lados de la casa de habitación, tipo de vías de acceso, presencia de focos de contaminación, presencia de andenes, presencia de antejardines, presencia de garajes, materiales de la fachada y tipo de material de la puerta principal. 
Teniendo en cuenta las anteriores variables se ha establecido la siguiente clasificación: estrato 1 bajo - bajo, estrato 2 bajo, estrato 3 medio -bajo, estrato 4 medio, estrato 5 medio - alto, estrato 6 alto. Ubicándose las poblaciones más pobres en los estratos 1 y 2 , y las población con mayores recursos económicos en los estratos 5 y 6.

La población de muestra fue elegida por ser este grupo de estudiantes parte de un proceso educativo, en el que se han venido implementando las tecnologías de la información y la comunicación de acuerdo con los requerimientos del Ministerio de Educación, dentro de los objetivos de política de calidad contemplados en el programa nacional de innovación educativa con el uso de las TIC que se implementó a partir del año 2008 y, además, de la presencia de su utilización en los diferentes entornos en que se vive en la actualidad. Por otra parte, porque los alumnos involucrados cuentan con experiencia sobre el uso de internet, especialmente en lo referente a la red social Facebook, de otro lado, porque se encuentran en una edad en la que pueden hacer una evaluación crítica de su proceso educativo y sugerir la implementación de nuevas estrategias para la construcción de conocimiento.

\section{La realidad de las interacciones de los estudiantes en Facebook}

El estudio analiza las actividades que se pueden desarrollar desde Facebook y para tal efecto se ha elegido el método cualitativo, puesto que la indagación apunta a reflexionar sobre la actitud de un grupo de seres humanos, en este caso, estudiantes, ante el surgimiento de las nuevas tecnologías y su uso en el desarrollo de las actividades escolares. Este método, según Vela (2004), "pone énfasis en la visión de los actores y el análisis" (p.9) involucrados en el desarrollo de la investigación, dando mayor importancia al significado de las relaciones entre las personas.

El desarrollo del análisis de los hallazgos de la aplicación de dos entrevistas semiestructuradas y de los datos registrados en las rejillas de observación, fueron organizados en tablas de resumen y tablas de incidencia para establecer la relación semántica a partir de su clasificación y codificación; de acuerdo con patrones dominantes para realizar su categorización, revisión y análisis de dominios (Spradley, 1980) o representaciones de la cultura que se subdividen en otras subcategorías, teniendo en cuenta que los elementos incluidos en una categoría tienen un significado en común.

De esta manera, para alcanzar el objetivo de analizar el uso de la red Facebook por parte de los estudiantes involucrados en la investigación, se aplican dos entrevistas, cada una con doce y diez preguntas, respectivamente, cuyo guion temático gira en torno al conocimiento de dicha red y sus diferentes usos; estas son realizadas de manera presencial en los salones de clase.

De igual manera se hacen dos sesiones de observaciones en los muros de algunos jóvenes, pero de estas solo participaron diecisiete alumnos, quienes voluntariamente agregan a 
la docente investigadora como contacto en la red en mención. De los ocho restantes, cinco afirman no ser usuarios y tres manifiestan abiertamente no querer ser objeto de las indagaciones en sus muros; estas se realizaron de manera virtual con el uso de una computadora, red de internet alámbrica y cuenta de Facebook, a través de la cual se accede para revisar qué herramientas utilizan los estudiantes y el tipo de actividades que desarrollan.

Una vez realizadas las entrevistas, sus respuestas se organizan por niveles de incidencia y con la técnica cromática, dando un color a cada respuesta. A continuación se hace el análisis de los datos a partir de la elaboración de tablas de resumen, de las que se seleccionan conceptos relevantes de acuerdo con los patrones dominantes o definiciones que incluyen nuevas subcategorías, teniendo en cuenta que los elementos incluidos en una categoría tienen un significado en común (Spradley,1980, citado en Rodríguez,1999), para realizar la categorización de elementos, tales como: término inclusivo o nombre de categoría y término incluido o categoría de menor rango, que se encuentra dentro de un dominio (Spradley, 1980), entre los que se establece una relación semántica.

Los datos de las rejillas de observación se organizan en tablas de resúmenes para establecer los patrones dominantes, teniendo en cuenta los mismos aspectos de las tablas de entrevistas. De los hallazgos obtenidos en las diferentes tablas se seleccionan los conceptos más relevantes de los resultados, a partir de los cuales se establecen las categorías y subcategorías para ser registrados en una tabla de incidencias que permita verificar su relación con los datos obtenidos.

De la aplicación de las entrevistas se establece que los veinticinco estudiantes tienen conocimientos básicos sobre la red en mención, pero solamente veintidós son usuarios, los tres restantes afirman haber usado sus herramientas en compañía de algunos amigos pero que no sienten interés por crear la cuenta. Además, sostienen que son muy pocas las veces que los estudiantes han utilizado Facebook en actividades escolares y que les gustaría que se implementara el uso de sus herramientas en los procesos académicos porque hasta ahora está prohibido su utilización en horas de clase. De la aplicación de las observaciones a los diecisiete estudiantes se concluye que los alumnos interactúan con frecuencia en Facebook en actividades como publicación de comentarios, envío de mensajes, participación de encuestas, compartir enlaces y publicación de fotos, entre otros. Se puede afirmar que el desarrollo de actividades escolares es mínimo con el uso de la red en mención.

Las metas de los estudiantes al usar Facebook pueden ser analizadas bajo la luz de los planteamientos teóricos de Goodiwn (1997), quien presenta una visión de la naturaleza social del lenguaje, como un sistema compartido de significados preestablecidos y una estructura que tiene consecuencias mucho más allá de la lingüística. Al explorar cómo este se utiliza en un proceso de comunicación -cuando se examina una situación de trabajo específico como por ejemplo las interacciones que realizan los alumnos con el uso de las herramientas 
de la red en mención-, se aprecia que existe coherencia entre los hallazgos y su afirmación acerca de que es necesario que los interlocutores -cuando usen un término adecuado a un contexto determinado, para este caso el desarrollo de actividades en medios virtuales a partir de internet-, logren comprender el mensaje que se da. Cabe agregar que entre la información compartida por los estudiantes se observa que tienen un conocimiento claro de los nuevos términos que surgen dentro de dicho contexto, pero que con el uso de este medio se evidencia una escasa relación con temáticas de tipo académico.

Dentro de los ambientes mediados por tecnología, la red social Facebook se ha convertido en una de las prácticas cotidianas de las personas, especialmente de los jóvenes, porque permite el intercambio de diversos tipos de información rompiendo fronteras de espacio de tiempo. Según Pardo (2010), este es "un sistema operativo social" (p. 83) que ofrece herramientas de utilidad para la construcción de conocimiento dentro de las prácticas educativas.

La primera categoría que se establece es la relacionada con el uso de la red social de Facebook por parte de los estudiantes de la institución educativa Héctor Julio Rangel Quintero. Vale la pena destacar que en la actualidad las tecnologías ofrecen nuevas formas de comunicación. James (2004) presenta una propuesta sobre una alternativa de aprendizaje, en la que se tienen en cuenta los intereses de los niños y jóvenes, como por ejemplo el uso de video juegos en los procesos de construcción de conocimiento; se destaca que dentro de las actividades que desarrollan los estudiantes se encuentran las interacciones y la utilización de redes sociales. Mediante los resultados del estudio se observa que en su mayoría son usuarios y argumentan que sería una buena estrategia para el desarrollo de actividades académicas, porque fomenta el proceso de aprendizaje como el producto de la participación social Fernández (2009a). Además, Wenger (1991) considera que "el significado del aprendizaje debe basarse en la experiencia de la persona que es concebida como miembro de una comunidad" (p.46). En estudios realizados por Chica (2009) se propone el uso de las aulas virtuales inteligentes como estrategia para alcanzar un proceso de formación, según los requerimientos del mundo globalizado destacando de esta manera la importancia de la tecnología en procesos de enseñanza aprendizaje.

Tanto los planteamientos de la perspectiva sociocultural de Vygotsky, como los presentados por La Casa (1988) en lo relacionado con el desarrollo cognitivo de las personas, prestan especial atención a la forma en que el niño va tomando conciencia de sí mismo a partir de sus experiencias con el medio y de su propia acción dentro de la interacción social, progresivamente con la utilización de distintos medios hasta llegar al uso de tecnologías entre las que se destaca el desarrollo de actividades con la utilización de Facebook. Teniendo en cuenta que en las observaciones realizadas en los muros de los estudiantes se puede detectar un continuo uso de sus herramientas en diferentes tipos de interacciones, como publicación de fotos, mensajes, comentarios y videos, etc. 
Dentro de las diferentes actividades por seguir en este estudio se tiene como referencia la teoría deontológica de Kant, la cual menciona que el último fin de las personas es lograr el bien de todos, y la consecuencialista de Jeremy Bentham: toda actuación tiene una consecuencia que puede afectar de manera directa e indirecta a los seres humanos. Mayan (2001) considera que su cumplimiento durante el desarrollo de las actividades se detecta a partir de la formulación de una pregunta en cuanto a su conocimiento y práctica. Además se pueden manifestar por el tipo de comentarios encontrados en los diferentes muros.

Sancho (2006) considera que las nuevas perspectivas de la educación a nivel interdisciplinar no son enfocadas como una crítica a la formación en un área determinada; dentro de los procesos de construcción de conocimiento se hace necesario poner las prácticas educativas al margen de las políticas de globalización cultural; además sostiene que en la actualidad se hace evidente la exigencia de otro tipo de preparación cognitiva, social y afectiva a partir de la activación de competencias que promuevan el desarrollo de nuevas destrezas; afirma que un paradigma educativo se da en procesos de transformación social dentro de la concepción cultural de la psicología. Teniendo en cuenta la anterior información se puede observar que con el uso de Facebook los estudiantes vienen incorporando y transformando nuevos conceptos disciplinares, a saber: fotos de perfil, comentarios en el muro, etiquetar, compartir, mensajes en el muro, comentarios, chat y me gusta, entre otros, los cuales han adquirido una nueva significación con su inserción en las interacciones que se dan a partir de Facebook.

Además, se puede destacar que la implementación de Facebook en actividades académicas se plantea como una buena estrategia que conlleva la inserción de nuevos modelos pedagógicos, especialmente aquellos que se han realizado basados en la perspectiva sociocultural o sociohistórica de Vigotsky. Así, es necesario tener de presente que en la actualidad la mayoría de los procesos comunicativos giran en torno al uso de las nuevas tecnologías y se debe brindar la posibilidad al estudiante de recibir orientación del conocimiento disciplinar en ambientes mediados por tecnología; a partir de la implementación de prácticas con el uso de herramientas como las de Facebook en el contexto educativo. Según Vásquez, Romero y Molina (2009) existen otras formas de crear ambientes pedagógicos con el uso de las TIC, tan efectivos como los comunes que llevan a fomentar la comunicación, la interactividad y la socialización.

Teniendo en cuenta la información anterior se plantea un nuevo rol de los actores educativos con el uso de Facebook en procesos de aprendizaje, pero a pesar de la gran cantidad de interacciones que se pueden evidenciar, dentro de los resultados encontrados en las entrevistas y observaciones se detectaron muy pocas actividades de tipo académico con la utilización de las herramientas objeto del estudio, ya que no se observa algún tipo de interacciones entre estudiantes y docentes. Se presenta una gran actividad entre los alumnos con sus amigos o familiares en los que cumplen 
roles de trasmisores de mensajes, objeto de encuesta en Facebook, centro de comentarios y papel de emisores y receptores de información a través del chat.

Los resultados obtenidos de la presente investigación fueron analizados a la luz de la teoría sociocultural de Vigotsky, la cual, a partir de los estudios de Fernández (2009a) propone la participación social como una forma de aprendizaje. En ese sentido, en el contexto de las redes sociales se encontró que los estudiantes que participaron en el estudio sí establecen relaciones por ese medio, ya que el $84 \%$ de ellos manifestó ser usuarios de Facebook y utilizar sus herramientas para compartir información de diferente tipo, por lo que se concluye que estos pueden ser utilizados o aprovechados para implementar procesos de construcción de conocimiento. Cuando se formula la pregunta en relación a si son o no usuarios de la red en mención, veintiuno de los veinticinco alumnos de los entrevistados responden que sí, con lo que se evidencia su gusto por compartir información con la utilización de este recurso.

Por otra parte, los resultados de la investigación son coherentes con las propuestas de Copertari (2009), quien presenta un nuevo sistema de apropiación de la información y el aprendizaje sin la presencia del profesor, mediante el uso de nuevos paradigmas educativos a partir de la tecnología digital; con los hallazgos se observa que las herramientas como el chat pueden establecer un efectivo puente de comunicación entre profesores y estudiantes, al igual que la creación de grupos para el desarrollo de foros y presentación de información a partir de enlaces, videos y documentos, entre otros; presentando de esta manera espacios diferentes al aula de clase sin la necesidad de la presencia física del maestro.

De igual manera, cabe destacar la utilidad de los aportes de los planteamientos de James (2004) en los que recomienda la apertura a espacios virtuales dentro de la cultura del aprendizaje para facilitar el desarrollo de actividades sin fronteras de espacio o de tiempo. Dentro de los resultados se encuentra que los estudiantes realizan dichas interacciones durante su tiempo libre y en lugares diferente a la institución educativa. Asimismo, se tiene en cuenta los aportes de Pinaya (2005) en lo concerniente a la necesidad de implementar una reforma educativa en las prácticas de aula, hacia una orientación constructivista, con mayor interacción entre los que participan quienes sirven de base para la propuesta que se presenta sobre el desarrollo de actividades con el uso de las herramientas de Facebook. Entre las respuestas de los estudiantes se puede ver claramente que manifiestan la necesidad de romper con la cotidianidad de los procesos escolares. Planteamientos que se ratifican con los resultados de las entrevistas a los veinticinco estudiantes quienes afirman estar de acuerdo con el uso de estas herramientas durante el desarrollo de las temáticas escolares. Argumentos que presentan coherencia con las reflexiones de Pardo (2010) en las que afirma que dicha red "según el ranking de Alexa de noviembre de 2009, ya ocupa el segundo lugar entre las plataformas más visitadas del mundo, por detrás de Google" (p. 84), razón por la que se hace necesario tener en cuenta los gustos y preferencias de 
los alumnos en los procesos de enseñanza aprendizaje.

Por lo anterior, se puede afirmar que fue adecuada la teoría a la práctica utilizada en el estudio, porque coinciden las experiencias de los estudiantes con los planteamientos de los autores que han realizado estudios bajo la luz de la teoría sociocultural de Vigotsky, dado que la mayor parte de la literatura revisada está relacionada con la socialización del conocimiento; más exactamente en lo referente a estudios que destacan el surgimiento de nuevas prácticas educativas en las que se tenga en cuenta el contexto en los procesos de construcción de conocimiento.

En el desarrollo de la indagación cobran importancia los conceptos de Vigotsky (citado en Daniels, 2003) porque proponen basar las actividades de enseñanza - aprendizaje en la experiencia de las personas, concibiéndolas como miembro de una comunidad en la que sufre un proceso de transformación a partir de sus interacciones. Además presenta el aprendizaje como una actividad continua de comunicación social y el conocimiento como la realización de enlaces significativos entre la vida escolar y la cotidiana, mediante actividades específicas de aprendizaje de los alumnos, de tal forma que estos logren establecer relaciones fuera del aula y puedan usar sus aprendizajes para mejorar las actividades que se dan en ella. Con lo cual consiguen comprender de manera más clara los contenidos escolares.

Además se tiene en cuenta la concepción de Well (citado por Daniels, 2003) en la que afir- ma que "conocer se define como la actividad intencional de individuos que como miembros de una comunidad, emplean y producen representaciones con el esfuerzo colectivo de comprender mejor su mundo y transformarlo" (Daniels, 2003, p. 148). Como se puede ver los estudiantes objeto del estudio mediante el uso de Facebook comparten información, que de ser utilizada en actividades académicas pueden fortalecer el desarrollo de valores y habilidades.

De igual manera, se destaca que desde la perspectiva sociocultural, se reconoce a los individuos como seres que aprenden de las relaciones entabladas con otras personas, entonces es importante tener en cuenta el uso de las redes sociales para facilitar su desarrollo. Considerando el aprendizaje planteado por Lave y Wenger (1991), en el que afirman que los seres humanos pertenecen a un sistema de interacciones dentro de comunidades, que son las que definen su identidad como hombre social con un compromiso de aprendizaje a nivel individual y colectivo en el contexto al que pertenece, en las observaciones realizadas en los muros de los diecisiete estudiantes que aceptaron la aplicación de la estrategia, se puede ver que a partir del uso de Facebook comparten información, enlaces, videos, etc. Con lo que se demuestra que dichas herramientas se puede implementar en actividades académicas para mejorar los procesos de construcción de conocimiento. $Y$ aunque es mínimo su uso en actividades escolares, pueden resultar un excelente recurso para construir conocimiento con la creación de grupos de estudio virtuales. 
Teniendo en cuenta las propuestas de Hine (2006), el cual considera una nueva concepción de la etnografía, dentro de los contextos virtuales se realiza un análisis del funcionamiento de la tecnología y sus principales características, a fin de observar lo que sucede en las experiencias diarias de los individuos a partir del uso de estos entornos.

En primera medida, se aplica la primera entrevista semiestructurada con el objetivo de indagar sobre el conocimiento y uso de las redes sociales por parte de los alumnos objeto del estudio; lo cual se hace de manera simultánea a los participantes en el salón de clase. Esta estrategia ofrece como resultado que todos los alumnos tienen conocimientos básicos sobre las redes sociales, aunque no todos las utilizan y existe una tendencia a confundirlas con otro tipo de servicios de internet como buscadores o programas.

En segundo lugar, se realiza la aplicación de la segunda entrevista con el propósito de averiguar específicamente aspectos relacionados con el conocimiento y uso de Facebook por parte de los estudiantes que conforman la población de muestra; con la que se pudo indagar sobre el conocimiento y uso de Facebook por parte de la población de muestra, teniendo de esta manera un mayor acercamiento hacia la respuesta de la pregunta de investigación, logrando establecer que todos tienen conocimientos básicos sobre la red en mención, pero solamente veintiuno son usuarios; los cuatro restantes afirman haber usado sus herramientas en compañía de algunos amigos pero que no sienten interés por el uso de sus herramientas.
De la misma entrevista se obtiene como resultado que son muy pocas las veces que los estudiantes han utilizado Facebook en actividades escolares, pero que les gustaría que se implementara el uso de sus herramientas en los procesos académicos porque hasta ahora está prohibido su uso en horas de clase. Calderón y Aparicio (2009) destacan la importancia de las innovaciones para mejorar la calidad en la educación de tal forma que se oriente a las personas en su adaptación a los cambios tecnológicos permanentes del contexto para lograr suplir aquellas necesidades que les permitan alcanzar el éxito dentro de la sociedad.

De la aplicación de las observaciones a los diecisiete estudiantes se concluye que los alumnos interactúan con frecuencia en Facebook en actividades como publicación de comentarios, envío de mensajes, participación de encuestas, compartir enlaces y publicación de fotos, entre otros. Asimismo se puede afirmar que el desarrollo de actividades escolares es mínimo con el uso de la red en mención.

Las políticas educativas colombianas apuntan a lograr que los estudiantes adquieran un aprendizaje que les sirva para la vida según lo establecido por el Ministerio de Educación de Colombia, en el programa Nacional de Innovación Educativa con el uso de las TIC (2008). Al respecto cabe aclarar que en el país se ha iniciado un proceso hacia la construcción de la sociedad del conocimiento, pero aún hace falta implementar verdaderas estrategias de apropiación de las tecnologías que se reviertan en iniciativas de impacto, como lo han hecho otros países. Por otra parte, la Ley 115 de 1994 
en lo relacionado con los fines de la educación establece la importancia de promover en la persona y en la sociedad habilidades para desarrollar actividades a partir de la adopción de la tecnología que se necesita en los procesos de progreso del país y que le permita al estudiante ingresar al sector productivo.

Es importante destacar que a pesar del uso de las tecnologías de la información y la comunicación en diferentes áreas del conocimiento, aún no se desarrollan actividades académicas con prácticas que involucren la utilización de las herramientas de Facebook en las mismas. Este estudio ofrece como alternativa la implementación del uso de la red social en los procesos de enseñanza aprendizaje, de tal forma que se tengan en cuenta los nuevos estilos de comunicación y las nuevas herramientas tecnológicas que los jóvenes utilizan.

Los aspectos que aún faltan ser investigados en esta línea están relacionados con el punto de vista de los profesores sobre la aplicación de estrategias que permitan el uso de nuevos paradigmas educativos, especialmente aquellos que se encuentran relacionados con la integración de medios virtuales en los procesos de enseñanza-aprendizaje en la institución educativa, dado que el presente estudio ofrece un panorama de las perspectivas de los estudiante al respecto, pero todavía queda pendiente indagar en torno al tema sobre ¿cuál es la opinión de los docentes con respecto a la utilización de la red social Facebook en los procesos de orientación de los saberes disciplinares, durante el desarrollo de las actividades académicas en la institución educativa Héctor Julio Rangel
Quintero del municipio de Floresta, Boyacá, Colombia?

Con los resultados de la anterior propuesta se tendría una base que permitiría sensibilizar a los maestros con miras a promover el desarrollo de proyectos que giren en torno a la pregunta referente a ¿cuáles son los resultados de la implementación de actividades con el uso de las herramientas de Facebook en los procesos de enseñanza aprendizaje en la institución educativa Héctor Julio Rangel Quintero del municipio de Floresta? De tal manera que con la inserción de los recursos de Facebook se pueda evaluar su utilidad, haciendo un comparativo entre el antes y el después, para detectar la conveniencia o no de su implementación en los ambientes educativos.

Por otra parte, queda el reto de concientizar a los padres de familia sobre la necesidad de ofrecer a los estudiantes el acceso a las nuevas tecnologías dentro de sus núcleos familiares, para que brinden la posibilidad a los alumnos de complementar sus actividades escolares con el uso de medios virtuales para aprovechar el tiempo libre dentro de los hogares de una manera flexible y motivadora. Atendiendo a la propuesta de James (2004), quien afirma que el aprendizaje debe estar conectado a las nuevas culturas y de esta manera, los estudiantes, sin importar su origen socioeconómico, puedan alcanzar el éxito en la escuela y en su competencia para desenvolverse dentro del mundo de la alta tecnología global, en el que la lingüística informativa con el uso de diferentes herramientas y actividades remplaza las antiguas formas de aprender por unas que 
permiten introducir otros contextos diferentes en el ambiente escolar, incluyendo el uso del ordenador y juegos de video del tal forma que se tenga en cuenta las preferencias, tanto de niños como de jóvenes, a fin de que ellos se sientan más comprometidos con su proceso de construcción de conocimiento.

\section{Referencias}

Bugeja, M. (2006). Heads Ups: Facing Facebook: Unless we reassess our high-tech priorities, issues of student insensitivity, indiscretion, and fabrication will consumeus. The Chronicle of higher education. [Versión electrónica]. Recuperado de http:/chronicle. com/article/facing-the-Facebook/46904

Calderón, R. y Aparicio, C. (2009). La innovación: su relación en la vinculación universidad-sociedad como elemento de desarrollo. Revista Interamericana de Investigación, Educación y Pedagogía,1, vol. 3, 65- 79. Recuperado de https://docs. google.com/viewer?a=v\&pid=explorer\&chr ome=true\&srcid=0BxOMtiQ6i-nLYmM4Nm VIM2MtNDdhYi00NGMxLThjMWQtZTdIYjh jMzEyNzBI\&hl=en_US

Cassany, D., Luna, M. y Zans, G. (1994). Enseñar lengua. España: GRAÓ de IRIF.

Chica, F. (2009). La dialéctica de la pregunta y la respuesta como estrategia de autorregulación en la construcción de un aula vir- tual inteligente. Revista Interamericana de Investigación, Educación y Pedagogía, 1, vol. 2, 45- 63. Recuperado de https://docs. google.com/viewer?a=v\&pid=explorer\&chr ome=true\&srcid=0BxOMtiQ6i-nLNGY3ODQ2OGYtMmEzMS00NjI1LThhN2QtODA3 NjUONmZIMThI\&hl=en_US

Asociación Colombiana para la Investigación en Ciencia y Tecnología (2003). Cognición situada y estrategias para el aprendizaje significativo. Revista Electrónica de Investigación Educativa, 002, vol. 5, 105-117. México: Universidad Autónoma de Baja California, Ensenada.

Cole, M. (2003). Psicología cultural: una disciplina del pasado y del futuro. Madrid: Morata.

Copertari, S., Trottini, A. y Contesti, J. (2009). Educación virtual y procesos metacognitivos: un nuevo paradigma de educación a distancia. XIII Congreso Internacional de Informática en la Educación, No. 1. Editorial Universitaria.

Corvi, R. (1996). Introduction to the thought of karl popper publisher: routledge (Translated by Camiller, P.). London and New York.

Daniels, H. (2003). Aplicaciones educativas de la teoría sociocultural y de la actividad. Vygotsky y la pedagogía. Barcelona: Paidós. 
Duart, J. (1999). Herramientas para la gestión de proyectos educativos con TIC. Innovar en Educación. Recuperado de http://www. oei.es/tic/INTEGRA_Herramientas.pdf.

Engeström, Y. (1999). Learning by expanding: ten years later. Recuperado de http://lchc. ucsd.edu/MCA/Paper/Engestrom/expanding/intro.htm

Fernández, J. (2004). The appropriation and mastery of cultural tools in computer supported collaborative literacy practices (Doctoral thesis). United Kingdom: The Open University.

Fernández, J. (2009a). Las tecnologías de la información y la comunicación desde la perspectiva de la psicología de la educación. J. Arévalo Zamudio y G. Rodríguez Blanco. México: Secretaría de Educación Pública/Dirección General de Materiales Educativos.

Fernández, J. (2009b). Aprendiendo a escribir juntos: multimodalidad, conocimiento y discurso. Monterrey: Comité Regional Norte de Cooperación con la Unesco/ Universidad Autónoma de Nuevo León.

Fernández, J. y Silveyra, M. (2010). Disciplinary knowledge and gesturing in communicative events: a comparative study between lessons using interactive whiteboards and traditional whiteboards in Mexican schools. Technology, Pedagogy and Education, 19(2), 173-193.

Fernández, J. (2011). Multimodalidad y calidad educativa: Los retos de la construcción de conocimiento disciplinar en ambientes mediados por tecnología digital. Ponencia presentada en el XII Encuentro Internacional Virtual Educa, México, D. F.

Freire, P. (2005). Pedagogía del oprimido. (Trad. Jorge Mellado) (2a . ed.). México: Siglo XXI Editores.

Giroux, S. y Tremblay, G. (2008). De la teoría a la práctica. Metodología de las ciencias humanas. (17-44). México: Fondo de Cultura Económica.

Goodwin, Ch. (1997). The blackness of black: color categories as situated practice. Recuperado de www.sscnet.ucla.edu/clic/ cgoodwin/97black.pdf.

Gundermann, H. (2004). El método de los estudios de caso. En M. L. Tarrés (Ed.). Observar, escuchar y comprender sobre la tradición cualitativa en la investigación social. 251-288. México: Flacso, Colegio de México, Miguel Ángel Porrúa.

Haspelmath, M., Koenig, E. y Oesterreicher, W. (2001). Language typology 
and language universals sprachtypologie und sprachlicheuniversalien:La typologie les universaux des languesetlinguistiques. New York: Walter de Gruyter\& Co. KG Publishers.

Hernández, F. (2002). Didáctica de las ciencias sociales, geografía e historia. Barcelona: Graó, de IRIF.

Hernández, R., Fernández, C. y Baptista, P. (2006). Metodología de la investigación (4ª . ed.). México: Editorial McGraw-Hill.

Hildreth, P. y Kimble, C. (2004). Knowledge networks innovation trough communities of de practice. London: Ide Group Publishing.

Hine, C. (2006). Virtual ethnography. Recuperado de http://www.cirst.uqam.ca/pcst3/PDF/ Communications/HINE.PDF.

Hota, K. (2000). Encyclopedia of new media and educational planning, vol. 5, 13-114. New Delhi: Sarup \& Sons.

Iglesias, M. y Sánchez, M. (2007). Diagnóstico e intervención didáctica del lenguaje escolar. España: Editorial Netbiblo.

James, P. (2004). Situated language and learning: a critique of traditional schooling. London: Routledge.
Kress,G., Jewitt, C., Ogborn, J. y Tsatsarelis, Ch. (2001). Multimodal teaching: Theretorics of the science classroom. London: Series Editors: Chris Candlin and Srikant Sarangi.

Kunh, T. (1962). La estructura de las revoluciones científicas. University of Chicago Press. Recuperado de http://www.marxists. org/reference/subject/philosophy/works/us/ kuhn.htm

Lacasa, P. (2002). Cultura y desarrollo. En P. Herranz Ibarra y P. Sierra García. Cultura y Desarrollo, (17-50). Madrid: UNED.

Lacasa, P. Villuendas D. (1988). Acción y representación en el niño: interacción social y aprendizaje. Madrid: Centro de Publicaciones del Ministerio de Educación y Ciencia.

Lave, J. y Wenger, E. (1991). Situated learning: legitimate peripheral participation. Cambridge: Cambridge University Press.

Leontiev, A. (1978-2000). The problem of activity and psychology (Chapter 3).En Activity, consciousness, and personality. Disponible en Marxists Internet Archive, Recuperado de http://www.marxists.org/archive/leontev/ works/1978/index.htm.. 
Lillis, T. y Kinney, C. (2003). Analising language a student work book. London Road: The Open University.

Maduro, R. y Rodríguez, J. (2008). Degustando el sabor de los datos cualitativos. Revista Electrónica Actualidades Investigativas en Educación, mayo-agosto, 1-22. Recuperado de http://redalyc.uaemex.mx/src/inicio/ ArtPdfRed.jsp?iCve=44713044005\&iCve Num=13044\#.

Martínez, R., Facundo, I., Hernández, M. y Hernández, S. (2008). Entornos sociales y percepción metodológica. México: Editorial Universitaria.

Mayan, M. (2001). Una introducción a los métodos cualitativos. Módulo de entrenamiento para estudiantes y profesionales. Qual Institute Press. International Institute for Qualitative Methodology. México: Universidad Autónoma Metropolitana, Iztapalapa, México.

Mina, L. (2004). Economía y desarrollo. Estratificación socioeconómica como instrumento de focalización, 1, vol. 3, 55-65. Universidad Autónoma de Colombia. Recuperado de http://www.fuac.edu.co/revista/III/III/ tres.pdf.

Ministerio de Educación Nacional República de Colombia (2008). Programa Nacional de Innovación Educativa con Uso de TIC. Programa estratégico para la competitividad. Ruta de apropiación de TIC en el Desarrollo Profesional Docente. Recuperado de http://castor.unab.edu.co/bbcswebdav/ courses/588-201112-MTEM/temas/unidad0/recursos/ruta_superior.pdf .

Moll, L. (1990). Instructional implications and applications of sociohistorical psychology. Cambridge, MA: Harvard University Press.

Moreno, A. (2005). Sociología de la educación: claves teóricas. España: Septem Ediciones.

Pardo, H. (2010). Geekonomía. Un radar para producir en el postdigitalismo. Collección Transmedia XXI. Laboratori de Mitjans Interactius. Barcelona: Ediciones de la Universitat de Barcelona.

Pinaya, B. (2005). Constructivismo y práctica de aula en Caracollo. Bolivia: Plural Editores.

Plourde, L. (2003). Constructivim and elementary preservice science teacher preparation: knowledge to aplication [version electrónica]. College Student Journal.

Rodríguez, S., Quiles, L. y Herrera, L. (2005). Teoría y práctica del análisis de datos cualitativos. Proceso general y criterios de calidad. Revista Internacional de Ciencias 
Sociales y Humanidades, SOCIOTAM [en línea], XV (julio-diciembre): Recuperado de http://redalyc.uaemex.mx/redalyc/src/ inicio/ArtPdfRed.jsp?iCve=65415209.

Salmerón, H., Rodríguez, S. y Gutiérrez C, C. (2010). Metodologías que optimizan la comunicación en entornos de aprendizaje virtual. Comunicar, 34, vol. XVII, 163-171.

Sánchez, R. (2004). La observación participante como escenario y configuración de la diversidad de significados. En M. L. Tarrés (Ed.). Observar, escuchar y comprender sobre la tradición cualitativa en la investigación social, 97-131. México: Flacso, Colegio de México, Miguel Ángel Porrúa.

Sancho, J. (2006). Tecnología para transformar la educación. Madrid: Ediciones Akal.

Sandoval, C. (2002). Investigación cualitativa. Bogotá, Colombia: ARFO Editores. Recuperado de http://es.scribd.com/ doc/52074924/20/La-propuesta-metodologica-de-la-etnografia

Sepúlveda, E. (2001). ¿Qué es aprendizaje expansivo? Universidad de La Frontera, Departamento de Educación. Chile: Temuco.

Seedhouse, P. (2009). The Interactional Architecture of the Language Classroom. Bellaterra: Journal of Teaching \& Learning
Language \& Literature, (1)1, 1-13. Recuperado de http://ojs.uab.cat/index.php/jt/3/ article/viewFile/32/1. Recuperado el 7 de abril de 2011.

Spradley, J. (1980). The Ethnographic Interview. Belmont, CA: Wadsworth Cengage Learning. Recuperado de https://www. yousendit.com/download/dkJwUXVqMG NubHI4dnc9PQ

Spradley, J. (1980). Participant Observation. Belmont, CA: Wadsworth Cengage Learning. Recuperado de https://www.yousendit.com/ download/dkJwUXVxZy9wcFh2Wmc9PQ

Suchman, L. (2007). Human-machine reconfigurations: plans and situated actions. New York: Cambridge University Press.

Tarrès, M. (2001). Observar, escuchar y comprender sobre la tradición cualitativa en la investigación social (1‥ ed.). México: Flacso.

Tusón, A. (2002). El análisis de la conversación: entre la estructura y el sentido. Estudios de Sociolingüística, 3(1), 133-153. Recuperado de http://www.google.com/ url?sa $=$ t\&source $=$ web\&cd $=3 \&$ ved $=0 C C Q$ QFjAC\&url=http\%3A\%2F\%2Fwww.sociolinguistica.uvigo.es\%2Fdescarga_gratis. asp \%3Fid\%3D63\&rct=j\&q=analisis $\% 20$ de\%20la\%20conversacion\&ei=EhpwTaee 
OYWasAOZk4TECw\&usg=AFQjCNGWDeBP9_4dnPcM5JqLsOGXOHe4A\&cad=rja Recuperado el 7 de abril de 2011

Vásquez, C., Romero, A. y Molina, R. (2009). Estrategias pedagógicas para la motivación lectora a través de un software de lectura interactiva. Revista Interamericana de Investigación, Educación y Pedagogía, 2, 19-30. Recuperado de https://docs.google. $\mathrm{com} / \mathrm{viewer}$ ?a=v\&pid=explorer\&chrome =true \&srcid=0bxomtiq6i-nlodq5ywynwmtmmzlyy00n2e4ltk1zgutmjy5mdhhmjz

Vela, F. (2004). Un acto metodológico básico de la investigación social: la entrevista cualitativa. En M. L. Tarrés (Ed.). Observar, escuchar y comprender. Sobre la tradición cualitativa en la investigación social, (pp. 63-95). México: FLACSO.
Vigotsky, L. (1978). El desarrollo de los procesos psicológicos superiores. Barcelona: Grijalbo.

Yuni, J. y Urbano, C. (2009). Técnicas para investigar: Recursos metodológicos para la preparación de proyectos de investigación. Editorial Brujas. Versión electrónica. recuperado de http://site.ebrary. com/lib/uvirtualeducacionsp/docdetail.ac tion? docid $=10353071 \& \mathrm{p} 00=$ instrument os $\% 20$ recolecci\%c3\%b3n\%20datos $\% 20$ investigaci\%c3\%b3n\%20cualitativa

Zeichner, K. y Gore, J. (1990). Teacher socialization. In W.R. Houston (Ed.). Handbook of research on teacher education: A project of the Association of Teacher Educators, (pp. 329-348). New York: Mac millan. 